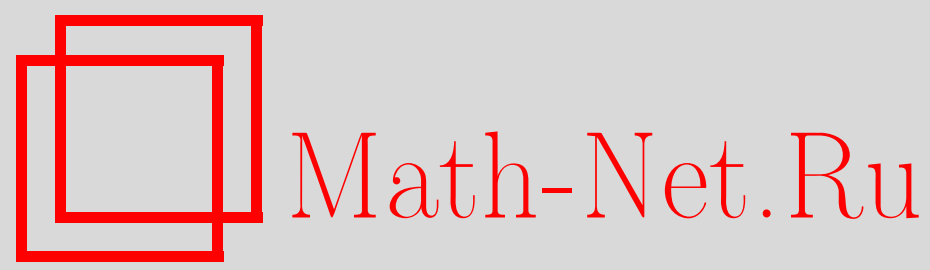

Е. П. Долженко, О граничном поведении компонент полианалитической функции, Матем. заметки, 1998, том 63, выпуск 6, 821-834

DOI: https://doi.org/10.4213/mzm1352

Использование Общероссийского математического портала Math-Net.Ru подразумевает, что вы прочитали и согласны с пользовательским соглашением http://www . mathnet.ru/rus/agreement

Параметры загрузки:

IP: 35.173 .137 .237

26 апреля 2023 г., 15:49:41

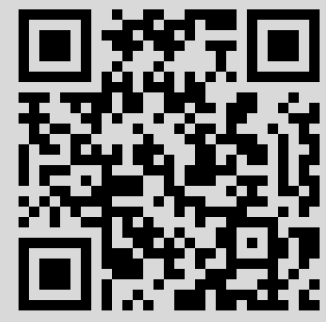




\section{О ГРАНИЧНОМ ПОВЕДЕНИИ КОМПОНЕНТ ПОЛИАНАЛИТИЧЕСКОЙ ФУНКЦИИ}

\section{Е.П. Долженко}

Для $p \geqslant 0$ вводятся двакласса: $C U^{p}(G)$-класс функций, равномерно непрерывных порядка $p$ в области $G \subset \mathbb{C}$, и $\mathfrak{M}^{p}(G)$ - класс функций, имеющих в $G$ ограниченность порядка $p$. Доказывается критерий принадлежности $n$-аналитической функции каждому из этих классов.

Библиографоия: 10 названий.

Введение. Функция $f(z)=f(x, y)$ (вообщеговоря, комплекснозначная), имеющая в области $G \subset \mathbb{C}$ непрерывные частные производные по $x$ и $y$ до порядка $n \geqslant 1$, назьвается полианалитической порядка $n$ в $G$, или $n$-аналитической в $G$, если она удовлетворяет обобщенному уравнению Коши-Римана $\partial^{n} f / \partial \bar{z}^{n}=0$. Класс всех таких функций обозначим через $A_{n}(G)$. Как обычно, класс всех непрерывных на $\bar{G}$ функций обозначим через $C(\bar{G})$. Функции $f \in A_{2}(G)$ назьваются бианалитическими в $G$. Всякую функцию $f \in A_{n}(G)$ можно единственным образом представить в виде

$$
f(z)=\varphi_{0}(z)+\bar{z} \varphi_{1}(z)+\cdots+\bar{z}^{n-1} \varphi_{n-1}(z),
$$

где функции $\varphi_{k}$ голоморфны в $G$. В единичном круге $D:=\{z:|z|<1\}$ представление (1) легко преобразуется к виду

$$
\begin{gathered}
f(z)=P(z, \bar{z})+g_{0}(z)+\left(1-|z|^{2}\right) g_{1}(z)+\cdots+\left(1-|z|^{2}\right)^{n-1} g_{n-1}(z), \quad z \in D \\
P(z, \bar{z})=\bar{z} P_{1}(z)+\cdots+\bar{z}^{n-1} P_{n-1}(z)
\end{gathered}
$$

где $P_{k}(z)$ при $k \geqslant 1-$ полином от $z$ степени $\leqslant k-1, g_{k}$ голоморфны в $D$.

Известно [1]-[3], что если замкнутая жорданова аналитическая кривая $Г$ ограничивает область $G \subset \mathbb{C}, w=\varphi(z)$ - какое-либо однолистное конформное отображение $G$ на круг $D, \psi$ - обратное отображение, то уравнение кривой $\Gamma$ может быть записано в виде $\bar{z}=a(z)$. Здесь функция

$$
a(z):=a_{\Gamma}(z)=\overline{\psi(1 / \overline{\varphi(z)})}
$$

Работа выполнена при финансовой поддержке Российского фонда фундаментальных исследований, грант № 96-01-01366. 
(нередко назьваемая функцией Шварца) голоморфна и однолистна в некоторой окрестности кривой $\Gamma$, причем в некоторой окрестности $Q\left(a_{\Gamma}\right)$ этой кривой вьполняется неравенство

$$
K \rho(z, \Gamma) \leqslant|\bar{z}-a(z)| \leqslant L \rho(z, \Gamma), \quad z \in Q\left(a_{\Gamma}\right),
$$

где $\rho(z, \Gamma)$ - расстояние от $z$ до $\Gamma$, а положительные постоянные $K$ и $L$ не зависят от $z \in Q\left(a_{\Gamma}\right)$. Отметим, что $a_{\partial D}(z)=1 / z$. Разложив $\bar{z}^{k}$ из $(1)$ по степеням $(\bar{z}-a(z))$, видим, что если функция $f(z) \quad n$-аналитична в некоторой, возможно односторонней $(Q \cap \Gamma=\varnothing)$, окрестности $Q$ аналитической кривой $Г$ и функция $a=a_{\Gamma}$ голоморфна в $Q$, то

$$
f(z)=h_{0}(z)+(\bar{z}-a(z)) h_{1}(z)+\cdots+(\bar{z}-a(z))^{n-1} h_{n-1}(z), \quad z \in Q,
$$

где функции $h_{k}$ голоморфны в $Q$. Это обобщение представления (2).

$\Phi$ ункции $\varphi_{k}, g_{k}$ и $h_{k}$ назьваются голоморфными компонентами функции $f \in A_{n}(G)$ (очевидно, $h_{n-1} \equiv \varphi_{n-1}$ ).

Известно, что "хорошие" свойства полианалитической функции не распространяются автоматически на ее голоморфные компоненты. Например, существуют функции $f$, бианалитические в $D$ и непрерьвные на $\bar{D}$, голомор фные компоненты которых не только имеют разрьв в каждой точке $\zeta \in \partial D$, но и почти всюду на $\partial D$ не имеют даже угловых пределов (см. лемму 5). Главным результатом этой работы является теорема 1 о том, что равномерные граничные свойства слагаемых в представлениях (2) и (5) функции $f \in A_{n}(G)$ не хуже, чем соответствуюшие свойства самой $f$.

Напомним и введем некоторые определения и обозначения.

Как обычно, $\omega(f, E, \delta):=\sup \{|f(z)-f(t)|: z, t \in E,|z-t| \leqslant \delta\}, \delta \geqslant 0,-$ модуль непрерьвности функции $f(z), z \in E \subset \mathbb{C}$. Функция $\omega(r)$ назьвается модулем непрерывности (вообще), если она определена, неотрицательна и не убывает при $r \geqslant 0$, причем $\omega(0)=0, \omega(u+v) \leqslant \omega(u)+\omega(v)$ при любых неотрицательных $u$ и $v$. Если $f(z)$ ограничена на выпуклом множестве $E$, то $\omega(r):=\omega(f, E, r)$ обладает всеми этими свойствами. Для любого модуля непрерывности $\omega(r)$ найдется выпуклый вверx при $r \geqslant 0$ и дважды непрерывно дифференцируемый при $r>0$ модуль непрерывности $\widetilde{\omega}(r)$ такой, что $3^{-1} \omega(r) \leqslant \widetilde{\omega}(r) \leqslant 3 \omega(r)$ при $r \geqslant 0$ (см. [4, п. 3.3]).

\section{1. Ореольная разность и ореольный модуль непрерывности.}

ОПРЕДЕЛЕНИЕ 1. Для числа $R>0$ и функции $f(z)$, заданной в точке $z$ и интегрируемой по Лебегу на окружности $\mathscr{C}=\{z:|t-z|=R\}$,

$$
\Delta_{R} f(x, y)=\Delta_{R} f(z):=\frac{1}{2 \pi R} \int_{\mathscr{C}}(f(t)-f(z))|d t|=\frac{1}{2 \pi} \int_{0}^{2 \pi}\left(f\left(z+R e^{i \vartheta}\right)-f(z)\right) d \vartheta
$$

назовем ореольной разностью радиуса $R$ функиии $f$ в точке $z$. Очевидно,

$$
\Delta_{R} f(z)=\frac{1}{2 \pi i} \int_{\mathscr{C}}(f(t)-f(z)) \frac{d t}{t-z} .
$$

Известно, что если функция $f(x, y)$ дважды гладкая вблизи $(x, y)$, то

$$
\lim _{R \rightarrow 0} R^{-2} \Delta_{R} f(x, y)=\frac{1}{4}\left(\frac{\partial^{2} f(x, y)}{\partial x^{2}}+\frac{\partial^{2} f(x, y)}{\partial y^{2}}\right)=\frac{\partial^{2} f(x, y)}{\partial z \partial \bar{z}}
$$


В случае функции $f(z)$, для которой в точке $z$ определена $n$-я степень оператора $\Delta_{R}$, выражение $\Delta_{R}^{n} f(z)$ будем назьвать $n$-й ореольной разностью радиуса $R$ функиии $f$ в mочке $z$.

Назовем ореольным модулем непрерывности порядка $q(q \geqslant 1)$ функции $f$, заданной в некоторой области $G$, функцию

$$
\omega_{\mathrm{or}}^{(q)}(f, G, \delta):=\sup \left\{\left|\Delta_{R}^{q} f(z)\right|\right\}, \quad 0<\delta<\infty, \quad \omega_{\mathrm{or}}^{(q)}(f, G, 0)=0,
$$

где супремум берется по всем парам $(z, R)$ таким, что $0<R \leqslant \delta$, при этом замкнутьй круг радиуса $q R$ с центром $z$ лежит в $G$.

ЗАмЕчАниЕ 1 . При $p \geqslant 0$ из определений $\Delta_{R}^{q} f(z)$ и $\omega_{\text {or }}^{(q)}(f, G, \delta)$ видно, что если $f \in \mathfrak{M}^{p}(G)$ (см. ниже определение 2), то $\omega_{\mathrm{or}}^{(q)}(f, G, \delta)=O\left(\delta^{s}\right)$ при $\delta \rightarrow 0$, где $s=\min \{p, q\}$, а если $f \in C U^{p}(G)$, то $\omega_{\text {or }}^{(q)}(f, G, \delta)=o\left(\delta^{s}\right)$.

Далее, как обычно,

$$
C_{k}^{p}:=\frac{k !}{p !(k-p) !} .
$$

ЛЕмма 1. Если $f \in A_{n}(G)$ представлена в виде (1), то

$$
\begin{gathered}
\Delta_{R} f(z)=\sum_{p=0}^{n-2} \bar{z}^{p} S_{p}(f, R ; z), \quad z \in G, \quad 0<R<\rho:=\rho(z, \partial G), \\
S_{p}(f, R ; z):=\sum_{k=p+1}^{n-1} C_{k}^{p} \frac{R^{2 k-2 p}}{(k-p) !} \varphi_{k}^{(k-p)}(z), \quad p=0, \ldots, n-2, \\
\Delta_{R}^{n-1} f(z)=(n-1) ! R^{2 n-2} \varphi_{n-1}^{(n-1)}(z), \quad n \geqslant 2, \quad z \in G, \quad 0<R<\frac{\rho(z, \partial G)}{n-1} .
\end{gathered}
$$

ДоКАЗАТЕЛЬСТВо. Если $\{t:|t-z| \leqslant R\} \subset G$, то

$$
\Delta_{R} f(z)=\sum_{k=1}^{n-1} \frac{1}{2 \pi i} \int_{\mathscr{C}} \frac{\bar{t}^{k}-\bar{z}^{k}}{t-z} \varphi_{k}(t) d t, \quad \mathscr{C}=\{z:|t-z|=R\}
$$

(см. (6) и (1)). Далее,

$$
\begin{gathered}
\bar{t}^{k}-\bar{z}^{k}=\sum_{p=0}^{k-1} C_{k}^{p}(\bar{t}-\bar{z})^{k-p} \bar{z}^{p} \\
\int_{\mathscr{C}}(\bar{t}-\bar{z})^{q} \varphi_{k}(t)(t-z)^{-1} d t=R^{2 q} \int_{\mathscr{C}} \varphi_{k}(t)(t-z)^{-q-1} d t=R^{2 q} \frac{2 \pi i}{q !} \varphi_{k}^{(q)}(z), \\
\frac{1}{2 \pi i} \int_{\mathscr{C}} \frac{\bar{t}^{k}-\bar{z}^{k}}{t-z} \varphi_{k}(t) d t=\sum_{p=0}^{k-1} C_{k}^{p} \frac{1}{(k-p) !} R^{2(k-p)} \bar{z}^{p} \varphi_{k}^{(k-p)}(z), \\
\Delta_{R} f(z)=\sum_{k=1}^{n-1} \sum_{p=0}^{k-1} C_{k}^{p} \frac{1}{(k-p) !} R^{2(k-p)} \bar{z}^{p} \varphi_{k}^{(k-p)}(z)=\sum_{p=0}^{n-2} \bar{z}^{p} S_{p}(f, R ; z)
\end{gathered}
$$

(см. (9)), и (7) доказано. Так как старший член (при $p=n-2, k=n-1)$ суммы (7) имеет вид $\bar{z}^{n-2} S_{n-2}(f, R ; z)=\bar{z}^{n-2}(n-1) R^{2} \varphi_{n-1}^{\prime}(z)$, для $(n-1)$-й степени оператора $\Delta_{R}$ получаем выражение (8). 
СлЕДСТВИЕ 1. Если $f \in A_{n}(G), n \geqslant 2, m o$ (cм. (1))

$$
\left|\varphi_{n-1}^{(n-1)}(z)\right| \leqslant C_{n} \rho^{2(1-n)} \omega_{\text {or }}^{(n-1)}\left(f, G, \frac{\rho}{n}\right), \quad z \in G, \quad \rho=\rho(z, \partial G),
$$

$2 \partial e$

$$
C_{n}=\frac{n^{2 n-2}}{(n-1) !}<e^{n} n^{n-1}(2 \pi(n-1))^{-1 / 2} .
$$

(Для оценки $C_{n}$ нужна формула Стирлинга и неравенство $(n /(n-1))^{n-1}<e$. )

2. Равномерная ограниченность и равномерная непрерывность. Пусть $p-$ действительное число. Обозначим через $\langle p\rangle$ наибольшее целое число строго меньшее $p$ $(p=\langle p\rangle+\alpha, 0<\alpha \leqslant 1)$.

ОПРЕДЕЛЕНИЕ 2. Ограниченными (соответственно равномерно непрерьвными) порядка 0 в области $G$ назовем функции, ограниченные (соответственно равномерно непрерьвные) в этой области. При $p>0$ назовем функцию $f(z)=f(x, y)$ ограниченной порядка $p$ в области $G$, если у нее в $G$ существуют все частные производные порядков не выше $\langle p\rangle$, причем производные порядка меньшего $\langle p\rangle$ удовлетворяют условию Lip 1 (считаем $\left.\partial^{0} f / \partial x^{0} \partial y^{0}:=f\right)$, а все производные порядка $\langle p\rangle-$ условию $\operatorname{Lip}(p-\langle p\rangle)$. Если при этом существуют все частные производные порядка $[p]$ и их модули непрерывности в $G$ суть величины порядка $o\left(r^{p-[p]}\right)$ при $r \rightarrow 0$, то назовем $f$ равномерно непрерывной порядка $p$ в области $G$. При $p<0$ назовем локально ограниченную в $G$ функцию $f$ ограниченной порядка $p$, если $|f(z)|=O\left(\rho^{p}\right)$ при $\rho=\rho(z, \partial G) \rightarrow 0$; если $f$ непрерывна в $G,|f(z)|=o\left(\rho^{p}\right)$ при $\rho \rightarrow 0$, то назовем $f$ равномерно непрерывной порядка $p$ в области $G$.

Очевидно, если две любых точки $z$ и $t$ ограниченной области $G$ можно соединить в $G$ некоторой гладкой кривой $L(z, t)$ длины $\leqslant C|z-t|$, где $C$ не зависит от $z$ и $t$, то ограниченность (равномерная непрерывность) целого порядка $p>0$ полианалитической в $G$ функции $f$ эквивалентна ограниченности (соответственно равномерной непрерьвности) в $G$ всех ее частных производных порядка $p$. Класс всех функций $f$, имеющих в области $G$ ограниченность порядка $p$, будем обозначать через $\mathfrak{M}^{p}(G)$, а имеюших равномерную непрерьвность порядка $p$ - через $C U^{p}(G)$. Обобщением классов $\mathfrak{M}^{p}(G)$ являются классы $M\left(r^{p} \omega(r), G\right)$.

ОПРЕДЕЛЕНИЕ 3 . Пусть $p$ - целое число, $p \in(-\infty, \infty), \omega(r)$ - модуль непрерьвности, $G$ - область, функция $f$ определена и локально ограничена в $G$. Скажем, что $f$ обладает ограниченностью уточненного порядка $r^{p} \omega(r)$, и будем писать $f \in M\left(r^{p} \omega(r), G\right)$, если для $p<0|f(z)|=O\left(\rho^{p} \omega(\rho)\right)$ при $\rho=\rho(z, \partial G) \rightarrow 0$ или для $p \geqslant 0 f$ имеет в $G$ частные производные всех порядков $\leqslant p$, причем модули непрерывности всех производных порядка $p$ в $G$ суть $O(\omega(r))$, а производные меньших порядков в $G$ удовлетворяют условию $\operatorname{Lip} 1$.

Согласно сказанному вьше для ограниченной области $G$ (а именно такие области рассматриваются) мы, не уменьшая общности, можем считать, что либо $\omega(r) \equiv 1$ при $r>0(\omega(0)=0)$, либо $\omega(r)$ непрерьвна и вьпукла вверх при $r \geqslant 0$ и непрерьвно диффференцируема при $r>0$. При этом, очевидно, в определениях классов $\mathfrak{M}(\cdot)$ и $C U(\cdot)$ можно не требовать того, чтобы функция $\omega(r)$ обладала свойствами модуля непрерьвности или отмеченными усиленными свойствами при всех $r \geqslant 0$; достаточно, чтобы эти свойства имели место на некотором отрезке $[0, \varepsilon], \varepsilon>0$ (в этом случае $\omega(r)$ можно продолжить на $[0, \infty)$ с сохранением указанных свойств). 
ЛЕмма 2. Пусть $G$-область, ограниченная конечным числом замкнутых гладких жордановых кривых без общих точек, $j, p(j>p, j \geqslant 1)$ - иелье числа, $\omega(r)-$ модуль непрерывности, функиия $f(z)=f(x, y)$ имеет в $G$ непрерывные частные производные всех порядков $\leqslant j$, при этом производные порядка $j$ принадлежат $M\left(r^{p-j} \omega(r)\right)$. Тогда

$$
\begin{gathered}
f \in M\left(r^{p} \omega(r)\right), \quad p \leqslant-2, \\
f \in M\left(\int_{r}^{1} \frac{\omega(s)}{s^{2}} d s\right), \quad p=-1, \\
f \in M\left(r^{p} \int_{r}^{1} \frac{\omega(s)}{s} d s\right), \quad p \geqslant 0, \\
f \in M\left(r^{p} \int_{0}^{r} \frac{\omega(s)}{s} d s+r^{p+1} \int_{r}^{1} \frac{\omega(s)}{s^{2}} d s\right), \quad p \geqslant 0, \quad \int_{0}^{1} \frac{\omega(s)}{s} d s<\infty, \\
f \in M\left(r^{p} \int_{0}^{r} \frac{\omega(s)}{s} d s\right), \quad p \geqslant 0, \quad j=p+1, \quad \int_{0}^{1} \frac{\omega(s)}{s} d s<\infty .
\end{gathered}
$$

ЗАмЕчАниЕ 2. Если $\omega(r)$ - выпукльй вверх и непрерывно дифференцируемьй при $r>0$ модуль непрерьвности, то соотношения (11)-(13) можно записать в "каноническом" виде $f \in M\left(r^{q} \omega_{k}(r)\right)$ (см. определение 3 ), где $q$ - целое число, а $\omega_{k}(r)$ - выпуклые вверх и непрерывно дифференцируемые при $r>0$ модули непрерывности $(k=1,2,3,4)$.

ДокАЗАТЕЛЬСТво ЛЕммы 2. Считаем функцию $\omega(r)$ вьпуклой вверх при $r \geqslant 0$ и непрерьвно дифференцируемой при $r>0$.

Пусть $L(z)$ - какая-либо гладкая кривая, соединяющая некоторую фиксированную точку $b \in G$ с $z \in G$,

$$
g_{j, q}(t):=\left.\frac{\partial^{j} f(z)}{\partial x^{q} \partial y^{j-q}}\right|_{z=t}, \quad 0 \leqslant q \leqslant j
$$

$d g_{j, q}(t) / d s$ - производная $g_{j, q}(t)$ по длине дуги кривой $L(z)$ в отрищательном направлении в точке $t \in L(z)$. Очевидно,

$$
\left|\frac{d g_{j-1, q}(t)}{d s}\right| \leqslant\left|g_{j, q+1}(t)\right|+\left|g_{j, q}(t)\right|, \quad t \in L(z), \quad 0 \leqslant q \leqslant j-1 .
$$

Выбирая кривую $L(z)$ так, чтобы ее конец приближался к $z$ по нормали к $\Gamma=\partial G$ (нормали из ближайшей к $z$ точки $\zeta \in \Gamma$ ), проходящей через $z$, и интегрируя $d g_{j-1, q}(t) / d s$ по $d s$ вдоль $L(z)$ от $z$ до $b$, получим равномерное по $z \rightarrow \Gamma$ соотношение

$$
\left|\frac{\partial^{j-1} f(z)}{\partial x^{q} \partial y^{j-1-q}}\right|=O\left(\int_{\rho}^{1} r^{p-j} \omega(r) d r\right), \quad \rho=\rho(z, \Gamma) \rightarrow 0, \quad 0 \leqslant q \leqslant j-1 .
$$

Последовательно применяя равенство (14) и заменяя в нем $j$ на $j-1, j-2, \ldots, 1$, с помощью формулы Коши для $j$-кратного повторного интеграла по нижнему пределу получим

$$
|f(z)|=O\left(\int_{\rho}^{1}(r-\rho)^{j-1} r^{p-j} \omega(r) d r\right)=O\left(\int_{\rho}^{1} r^{p-1} \omega(r) d r\right), \quad \rho=\rho(z, \Gamma) \rightarrow 0 .
$$


При $p=-1$ это дает (11). При $p \leqslant-2$, используя формулу интегрирования по частям и то, что при $u(r):=\int_{0}^{r} \omega(x) d x \frac{1}{2} r \omega(r) \leqslant u(r) \leqslant r \omega(r)$, получаем неравенство

$J:=\int_{\rho}^{1} r^{p-1} \omega(r) d r=u(1)-\rho^{p-1} u(\rho)+(1-p) \int_{\rho}^{1} u(r) r^{p-2} d r>0-\rho^{p} \omega(\rho)+\frac{1}{2}(1-p) J$,

откуда $J<2 \rho^{p} \omega(\rho)$. Вместе с (15) это дает (10).

Если $p \geqslant 0$, то, последовательно применяя равенство (14) и заменяя в нем $j$ на $j, j-1$, $j-2, \ldots, p+1$, получим

$$
\left|\frac{\partial^{p} f(z)}{\partial x^{q} \partial y^{p-q}}\right|=O\left(\int_{\rho}^{1} \frac{\omega(r)}{r} d r\right), \quad \rho=\rho(z, \Gamma) \rightarrow 0, \quad 0 \leqslant q \leqslant p,
$$

что доказьвает (12).

Наконец, пусть $p \geqslant 0$ и $\int_{0}^{1} \frac{\omega(s)}{s} d s<\infty$. Покажем, что каждая функция

$$
g_{p, q}(z)=\frac{\partial^{p} f(z)}{\partial x^{q} \partial y^{p-q}}, \quad 0 \leqslant q \leqslant p
$$

непрерьвно продолжается на Г и

$$
\omega\left(g_{p, q}, \Gamma, \delta\right)=O\left(\int_{0}^{\delta} \frac{\omega(r)}{r} d r+\delta \int_{\delta}^{1} \frac{\omega(r)}{r^{2}} d r\right), \quad \delta \rightarrow 0
$$

причем в случае $j=p+1$ второе слагаемое из правой части (16) отсутствует.

Заметим, что если $j=p+1$, то

$$
g_{p+1, q}(z)=O\left(\frac{\omega(\rho)}{\rho}\right), \quad \rho=\rho(z, \Gamma) \rightarrow 0, \quad 0 \leqslant q \leqslant p+1,
$$

так как $g_{j, q} \in M\left(r^{p-j} \omega(r)\right)$. Если $j>p+1$, то, как выше, последовательно применяя равенство $(14)$ и заменяя в нем $j$ на $j, j-1, j-2, \ldots, j-p-1$, получим при $0 \leqslant q \leqslant p+1$

$$
\left|g_{p+1, q}(z)\right|=O\left(\int_{\rho}^{1}(r-\rho)^{j-p-2} r^{p-j} \omega(r) d r\right)=O\left(\int_{\rho}^{1} \frac{\omega(r)}{r^{2}} d r\right), \quad \rho \rightarrow 0
$$

Пусть теперь $w \in \Gamma$, гладкая кривая $L(w)$ всеми своими точками, кроме $w$, лежит в $G$, начинается в точке $b \in G$ и оканчивается отрезком нормали к $\Gamma$ в точке $w$. Тогда ввиду (17) и (18) интеграл вдоль $L(w)$ по $d s$ от $d g_{p, q}(z) / d s$ существует и не зависит от выбора $L(w)$, так что $g_{p, q}(z)$ имеет в точке $w$ некоторый предел $g_{p, q}(w)$ по нормали. Если $v, w \in \Gamma$ и $d:=|v-w|$ мало́, то внутренние нормали длины $d$ к $\Gamma$, выходящие из $v$ и $w$, с концами $V$ и $W$ соответственно вместе с отрезком $[V, W]$ образуют трехзвенную ломаную, соединяющую $v$ с $w$. Из (18) следует, что при $j>p+1$ приращения $\Delta_{v V}$ и $\Delta_{w W}$ функции $g_{p, q}(z)$ на $[v, V]$ и $[W, w]$ суть величины порядка

$$
O\left(\int_{0}^{d}\left(\int_{\rho}^{1} \frac{\omega(r)}{r^{2}} d r\right) d \rho\right)=O\left(d \int_{d}^{1} \frac{\omega(r)}{r^{2}} d r+\int_{0}^{d} \frac{\omega(\rho)}{\rho} d \rho\right)
$$


а ее приращение $\Delta_{V W}$ на $[V, W]$ - величина порядка

$$
O\left(d \int_{d}^{1} \frac{\omega(r)}{r^{2}} d r\right)
$$

равномерно по парам $v$ и $w$ при $d \rightarrow 0$. Если $j=p+1$, то из (17) имеем

$$
\begin{gathered}
\Delta_{v V}=O\left(\int_{0}^{d} \frac{\omega(r)}{r} d r\right), \quad \Delta_{w W}=O\left(\int_{0}^{d} \frac{\omega(r)}{r} d r\right), \\
\Delta_{V W}=O(\omega(d))=O\left(\int_{0}^{d} \frac{\omega(r)}{r} d r\right) .
\end{gathered}
$$

Последнее порядковое равенство следует (ввиду вьпуклости вверх функции $\omega(r))$ из монотонного убывания $\omega(r) / r$. Таким образом, (16) доказано.

Далее, в случае односвязной области $G$ положим $h=1$, в случае неодносвязной $h:=\min \left\{1, h^{\prime}\right\}$, где $h^{\prime}$ - минимум попарных расстояний между связными компонентами Г. Пусть $\gamma$ - одна из таких компонент, $G(\gamma):=\{z \in G: \rho(z, \gamma)<h / 4\}$. Возьмем какие-либо точки $V, W \in G(\gamma)$. Пусть $v$ и $w$ - те точки на $\gamma$, ближайшие к $V$ и $W$ соответственно, расстояние между которыми минимально. Положим $\delta:=|V-W|$, $R:=\min \{\rho(V, \Gamma), \rho(W, \Gamma)\}$.

Если $\delta \geqslant R$, то $\left|\Delta_{V W}\right| \leqslant\left|\Delta_{v V}\right|+\left|\Delta_{v w}\right|+\left|\Delta_{w W}\right|$, так что

$$
\left|g_{p, q}(V)-g_{p, q}(W)\right| \leqslant B\left(\int_{0}^{\delta} \frac{\omega(r)}{r} d r+\delta \int_{\delta}^{1} \frac{\omega(r)}{r^{2}} d r\right), \quad \delta \geqslant 0, \quad 0 \leqslant q \leqslant p
$$

( $B$ не зависит от $V$ и $W$ ). Если $\delta<R$, то любая точка $t \in[V, W]$ удалена от $\Gamma$ не менее чем на $R / 2$, и из (17) при $j=p+1$ получаем

$$
\left|g_{p, q}(V)-g_{p, q}(W)\right| \leqslant C_{1}\left(\frac{2}{R}\right) \omega\left(\frac{R}{2}\right) \delta \leqslant C_{2} \int_{0}^{\delta} \frac{\omega(r)}{r} d r
$$

поскольку функция $\omega(r) / r$ убывает и

$$
\frac{2}{R} \omega\left(\frac{\delta}{2}\right) \delta \leqslant 2 \omega\left(\frac{\delta}{2}\right) \leqslant 2 \omega(\delta) \leqslant 2 \int_{0}^{\delta} \frac{\omega(r)}{r} d r
$$

При $j>p+1$ из (18) имеем

$$
\left|g_{p, q}(V)-g_{p, q}(W)\right| \leqslant C_{3}\left(\int_{0}^{\delta} \frac{\omega(r)}{r} d r+\delta \int_{\delta}^{1} \frac{\omega(r)}{r^{2}} d r\right)
$$

так что при $\delta=|V-W| \rightarrow 0$ и любом расположении точек $V$ и $W$ в $G(\gamma)$ имеем неравенство (19). Таким образом, соотношения (13) доказаны.

Из леммы 2 непосредственно вытекает необходимая для дальнейшего 
ЛЕмма 3. Если область $G$ ограничена конечным числом замкнутых гладких жордановых кривых без общих точек, $p$ - отрицательное или нечелое полоэсительное число, $j$ - натуральное число, все частные производные порядка $j$ функиии $f$ принадлежат классу $\mathfrak{M}^{p-j}(G)\left(\right.$ или $\left.C U^{p-j}(G)\right)$, mо $f \in \mathfrak{M}^{p}(G)$ (coответственно $\left.f \in C U^{p}(G)\right)$.

3. Основная теорема. Следующее утверждение является частным случаем теоремы 1 .

ЛЕммА 4. Пусть Г- - амкнутая жсорданова аналитическая кривая, функиия $a(z)=a_{\Gamma}(z)$ голоморфна и удовлетворяет неравенствам (4) на замыкании некоторой двусвязной области $Q$, ограниченной кривой $Г$ и замкнутой жордановой аналитической кривой $\mathscr{L}, \mathscr{L} \cap \Gamma=\varnothing$, функиия $h(z)$ голоморфна на $Q \cup \mathscr{L}, p-$ действительное число, $k$ - натуральное. Тогда для принадлежсности $(k+1)$-аналитической в $Q$ функиии $f(z)=(\bar{z}-a(z))^{k} h(z) \kappa \mathfrak{M}^{k+p}(Q)$ или $C U^{k+p}(Q)$ необходима и достаточна принадлежность $h \kappa \mathfrak{M}^{p}(Q)$ или $C U^{p}(Q)$ соответственно. Если $h \in \mathfrak{M}^{p}(Q)$ и $p+k>0$, то функиия $f$, доопределенная на $\Gamma$ нулем, и все ее частные производные порядков меньиих $k+\langle p\rangle$ удовлетворяют на $\bar{Q}$ условию Lip 1 , а производные порядка $k+\langle p\rangle-$ условию $\operatorname{Lip}(p-\langle p\rangle) ;$ при этом все производные порядков $0<q<k-p^{-}$равны 0 на $\Gamma\left(p^{-}:=\max \{-p, 0\}\right)$.

ДокАЗАтЕЛЬСтво. Далее $\rho:=\rho(z, \Gamma)<\rho(\Gamma, \mathscr{L}) / 2$.

Необходимость. Пусть $f \in \mathfrak{M}^{k+p}(Q)$. Если $p \geqslant 0$, то голоморфная функция

$$
h=\frac{1}{k !} \frac{\partial^{k} f}{\partial \bar{z}^{k}}
$$

принадлежит $\mathfrak{M}^{p}(Q)$, что и требуется. Если $p<0$ и $k+p \leqslant 0$, то $|f(z)|=O\left(\rho^{k+p}\right)$, $\rho \rightarrow 0$, и (из определения $f)|h(z)|=O\left(\rho^{p}\right), h \in \mathfrak{M}^{p}(Q)$. Если $p<0, k+p>0$, то в силу замечания 1 и следствия 1 имеем

$$
\left|h^{(k)}(z)\right|=O\left(\rho^{-2 k} \rho^{k+p}\right)=O\left(\rho^{p-k}\right), \quad h^{(k)} \in \mathfrak{M}^{p-k}(Q)
$$

$\left(h_{n} \equiv \varphi_{n}\right.$, см. (1) и (5)). Отсюда согласно лемме $3 h \in \mathfrak{M}^{p}(Q)$.

Достаточность. Так как

$$
\frac{\partial f}{\partial x}=\frac{\partial f}{\partial z}+\frac{\partial f}{\partial \bar{z}}, \quad \frac{\partial f}{\partial y}=i \frac{\partial f}{\partial z}-i \frac{\partial f}{\partial \bar{z}},
$$

TO

$$
\frac{\partial^{q} f(z)}{\partial x^{r} \partial y^{q-r}}=\sum_{\substack{0 \leqslant m \leqslant k \\ 0 \leqslant j \leqslant q \\ 1 \leqslant j+m \leqslant q}} u_{m, j}(z)(\bar{z}-a(z))^{k-m} h^{(j)}(z), \quad q \geqslant 1, \quad 0 \leqslant r \leqslant q,
$$

где функции $u_{m, j}(z)=u_{m, j}(q, r ; z)$ голоморфны на $Q \cup \Gamma$. Пусть $h \in \mathfrak{M}^{p}(Q)$.

1) Пусть $p>0$ - нецелое, $p=v+\alpha(v=[p] \geqslant 0,0<\alpha<1)$. Если $j \leqslant v$, то функция $f_{m, j}(z):=(\bar{z}-a(z))^{k-m} h^{(j)}(z)$ непрерьвна и удовлетворяет условию $\operatorname{Lip} \alpha$ на $\bar{Q}$ (см. (4)), и если $q<k$, то $f_{m, j}(z) \rightarrow 0$ при $\rho \rightarrow 0$. Если $j \geqslant v+1(>p)$, представим 
в $Q$ функцию $h^{(v)} \in \operatorname{Lip} \alpha$ в виде интеграла Коши по $\partial Q$. С помошью формулы Коши для производных получим соотношение

$$
h^{(j)}(z)=\frac{d^{j-v} h^{(v)}(z)}{d z^{j-v}}=O\left(\rho^{\alpha-(j-v)}\right)=O\left(\rho^{p-j}\right),
$$

которое вместе с (4) дает

$$
f_{m, j}(z)=O\left(\rho^{(k+p)-(m+j)}\right)=O\left(\rho^{k+p-q}\right),
$$

так что и в этом случае $f_{m, j}(z) \rightarrow 0$ при $q<k+p$ и $\rho \rightarrow 0$. Из сказанного следует, что

$$
\frac{\partial^{q} f(z)}{\partial x^{r} \partial y^{q-r}} \rightarrow 0 \quad \text { при } q<k, \quad 0 \leqslant r \leqslant q, \quad \rho \rightarrow 0 .
$$

Из $(21)$ имеем при $0 \leqslant r \leqslant q$ и $\rho \rightarrow 0$ соотношения (см. лемму 3 )

$$
\frac{\partial^{k+v+1} f(z)}{\partial x^{r} \partial y^{k+v+1-r}}=O\left(\rho^{\alpha-1}\right), \quad f \in \mathfrak{M}^{k+p}(Q)
$$

2) Пусть $p \geqslant 0$ - целое. Тогда $\left|h^{(j)}(z)\right| \leqslant M=$ const при $j \leqslant p, z \in Q$. Если $j>p$, $z \in Q$, то

$$
h^{(j)}(z)=\frac{1}{2 \pi i}(j-p) ! \int_{\mathscr{C}} h^{(p)}(t)(t-z)^{-(j-p)-1} d t, \quad \mathscr{C}=\left\{t:|t-z|=\frac{\rho}{2}\right\},
$$

откуда $\left|h^{(j)}(z)\right|=O\left(\rho^{p-j}\right)$. Итак, в любом случае выполняется равенство $(21)$, из которого следует ограниченность всех $(k+p)$-х частных производных функции $f$ и принадлежность $f$ к $\mathfrak{M}^{p+k}(Q)$, а также стремление к 0 (при $\rho \rightarrow 0$ ) всех ее частных производных порядков $q<k$.

3) Пусть $p<0, k+p \leqslant 0$. Тогда $|h(z)|=O\left(\rho^{p}\right),|f(z)|=O\left(\rho^{k+p}\right), f \in \mathfrak{M}^{k+p}(Q)$.

4) Наконец, пусть $p<0, k+p>0$. Из неравенства Коши

$$
\left|h^{(j)}(z)\right| \leqslant j ! \max \left\{|h(t)|:|t-z|=\frac{\rho}{2}\right\}\left(\frac{2}{\rho}\right)^{j}
$$

имеем $h^{(j)}(z)=O\left(\rho^{p-j}\right)$ при $\rho \rightarrow 0$. Отсюда и из (4) вытекает $(21)$. При $q=k+\langle p\rangle+1$ из $(21)$ и $(20)$ имеем

$$
\frac{\partial^{q} f(z)}{\partial x^{r} \partial y^{q-r}}=O\left(\rho^{p-\langle p\rangle-1}\right), \quad 0 \leqslant r \leqslant q .
$$

При целом $p$ это дает ограниченность производных порядка $q=k+\langle p\rangle+1=k+p$ функции $f$, а при нецелом $p$ означает, что производные порядка $q=k+[p]+1$ функции $f$ суть $O\left(\rho^{p-[p]-1}\right)$. В обоих случаях $f \in \mathfrak{M}^{k+p}(Q)$ (см. лемму 3$)$. Из $(21)$ также следует, что

$$
\frac{\partial^{q} f(z)}{\partial x^{r} \partial y^{q-r}} \rightarrow 0 \quad \text { при } \quad 0 \leqslant q<k+p, \quad 0 \leqslant r \leqslant q, \quad z \rightarrow \Gamma .
$$

Случай $h \in C U^{p}(Q)$ рассматривается совершенно аналогично.

Следующая теорема является основной в этой работе. Из нее следует, что слагаемые представлений (2) и (5) почти не интерферируют, т.е. они как бы “ортогональны”. Вероятно, этот факт играет важную роль в граничном поведении полианалитических функций. 
ТЕОРема 1. Пусть $G$ - область, в состав гранииы которой входит замкнутая жорданова аналитическая кривая $\Gamma, \rho(\Gamma, \partial G \backslash \Gamma)>0$, полианалитическая в $G$ функиия $f$ представлена вблизи Г в виде $(5), f_{k}(z):=(\bar{z}-a(z))^{k} h_{k}(z), 0 \leqslant k \leqslant n-1$. Тогда в некоторой односторонней окрестности $Q \subset G$ кривой $\Gamma$ имеем при любом $p \in \mathbb{R}$ әквивалениии:

$$
\begin{aligned}
f \in \mathfrak{M}^{p}(Q) & \Longleftrightarrow f_{k} \in \mathfrak{M}^{p}(Q) \quad \forall k, 0 \leqslant k \leqslant n-1 \\
& \Longleftrightarrow h_{k} \in \mathfrak{M}^{p-k}(Q) \quad \forall k, 0 \leqslant k \leqslant n-1, \\
f \in C U^{p}(Q) & \Longleftrightarrow f_{k} \in C U^{p}(Q) \quad \forall k, 0 \leqslant k \leqslant n-1 \\
& \Longleftrightarrow h_{k} \in C U^{p-k}(Q) \quad \forall k, 0 \leqslant k \leqslant n-1 .
\end{aligned}
$$

ДокАЗАТЕЛЬСтво. Пусть $Q, Q \subset G,-$ какая-либо односторонняя окрестность кривой $\Gamma$, в которой определена $a_{\Gamma}(z)$ и вьполняются неравенства $(4), \rho:=\rho(z, \Gamma)$.

Докажем (22). Пусть $f \in \mathfrak{M}^{p}(Q)$. Если $p \geqslant n-1$, то

$$
(n-1) ! h_{n-1}=\frac{\partial^{n-1} f}{\partial \bar{z}^{n-1}} \in \mathfrak{M}^{p-(n-1)}
$$

и $f_{n-1} \in \mathfrak{M}^{p}(Q)$ в силу леммы 4 . Если $p<n-1$, то

$$
\left|h_{n-1}^{(n-1)}(z)\right|=O\left(\rho^{p-2(n-1)}\right)
$$

согласно следствию 1. Так как $p<n-1$, то, интегрируя $h_{n-1}^{(n-1)}(z)(n-1)$-кратно, получим $h_{n-1} \in \mathfrak{M}^{p-(n-1)}(Q)$, откуда $f_{n-1} \in \mathfrak{M}^{p}(Q)$ ввиду леммы 4 . Таким образом,

$$
f \in \mathfrak{M}^{p}(Q) \Longrightarrow f_{n-1} \in \mathfrak{M}^{p}(Q)
$$

Поэтому и $(n-1)$-аналитическая функция $f(z)-(\bar{z}-a(z))^{n-1} h_{n-1}(z)$ принадлежит классу $\mathfrak{M}^{p}(Q)$. По доказанному функция $(\bar{z}-a(z))^{n-2} h_{n-2}(z)$ принадлежит $\mathfrak{M}^{p}(Q)$ и т.д., пока не установим, что $h_{0} \in \mathfrak{M}^{p}(Q)$. Итак,

$$
f \in \mathfrak{M}^{p}(Q) \Longrightarrow f_{k} \in \mathfrak{M}^{p}(Q) \quad \forall k, 0 \leqslant k \leqslant n-1
$$

Обратное очевидно. Эквиваленция $f_{k} \in \mathfrak{M}^{p}(Q) \Longleftrightarrow h_{k} \in \mathfrak{M}^{p-k}(Q)$ следует из леммы 4. Аналогично доказывается (23).

СлЕДСТВИЕ 2. Если область $G \subset \mathbb{C}$ ограничена конечным числом простых аналитических контуров без общих точек, $f \in A_{n}(G) \cap \mathfrak{M}^{p}(G)\left(\right.$ или $f \in A_{n}(G) \cap$ $\left.C U^{p}(G)\right), p \in \mathbb{R}$, mо (cм. (1)) $\varphi_{k} \in \mathfrak{M}^{p-n+1}(G), 0 \leqslant k \leqslant n-1$ (соответственно $\left.\varphi_{k} \in C U^{p-n+1}(G), 0 \leqslant k \leqslant n-1\right)$. Это утверждение нельзя усилить ни при каком наборе чисел $n \geqslant 1, p \in \mathbb{R}, 0 \leqslant k \leqslant n-1$.

Действительно, первая часть утверждения следует из теоремы 1. Для доказательства второй части возьмем $f(z)=(1-z \bar{z})^{n-1} g(z)$, где $g(z)$ голоморфна в $D, g \in \mathfrak{M}^{q}(D)$ при $q=p-n+1$, но $g \notin \mathfrak{M}^{q}(D)$ при $q>p-n+1$. 
СЛЕДСТВИЕ 3. Если область $G \subset \mathbb{C}$ ограничена простым аналитическим контуром $\Gamma, f \in A_{n}(G) \cap C(\bar{G})$, то в представлении (5) функиии $f$ имеет место равенство $h_{0}(t) \equiv f(t)$ на $Г$. Если при этом $h_{0}(z)$ голоморфна всюду в $G$, то $\omega\left(h_{0}, \bar{G}, \delta\right) \leqslant 108 \omega(f, \Gamma, \delta), \delta \geqslant 0$.

Действительно, $f \in C U^{0}(G)$, значит $h_{k} \in C U^{-k}(G), h_{k}(z)=o\left(\rho^{-k}\right)$ при $1 \leqslant k \leqslant n-1$ $(\rho=\rho(z, \Gamma) \rightarrow 0), f_{k}(z):=(\bar{z}-a(z))^{k} h_{k}(z) \rightarrow 0$ при $z \rightarrow \Gamma$, так что $f_{k}(t) \equiv 0$ при $k \geqslant 1, h_{0}(t) \equiv f(t)(t \in \Gamma)$. Неравенство $\omega(g, \bar{G}, \delta) \leqslant 108 \omega(g, \Gamma, \delta)$ доказано в [5] для произвольных голоморфных в $G$ функций $g \in C(\bar{G})$.

СлЕДСТВИЕ 4. Пусть функиия $f \in A_{n}(D)$ представлена в виде $(2), f_{k}(z):=$ $\left(1-|z|^{2}\right)^{k} g_{k}(z)$. Тогда

$$
\begin{aligned}
f \in \mathfrak{M}^{p}(D) & \Longleftrightarrow f_{k} \in \mathfrak{M}^{p}(D) \quad \forall k, 0 \leqslant k \leqslant n-1 \\
& \Longleftrightarrow g_{k} \in \mathfrak{M}^{p-k}(D) \quad \forall k, 0 \leqslant k \leqslant n-1, \\
f \in C U^{p}(D) & \Longleftrightarrow f_{k} \in C U^{p}(D) \quad \forall k, 0 \leqslant k \leqslant n-1 \\
& \Longleftrightarrow g_{k} \in C U^{p-k}(D) \quad \forall k, 0 \leqslant k \leqslant n-1 .
\end{aligned}
$$

СлЕдСТВИЕ 5. Если бианалитическая в круге $D$ функиия $f(z)=\varphi_{0}(z)+\bar{z} \varphi_{1}(z)$ удовлетворяет условию

$$
\omega(f, D, \delta)=O(\delta), \quad \delta \rightarrow 0
$$

(m.e. $f \in \operatorname{Lip} 1$ в $D)$, то ее голоморфные компоненты $\varphi_{0}(z)$ и $\varphi_{1}(z)$ имеют почти всюду на дD конечные угловые предель. Условие (24) нельзя ослабить до $\omega(f, G, \delta)=O(\omega(\delta)), \delta \rightarrow 0$, каков бы ни был нелипиичев (т.е. $\omega(\delta) / \delta \rightarrow \infty$ при $\delta \rightarrow 0)$ модуль непрерывности $\omega(\delta)$.

Последнее вытекает из следующего утверждения.

ЛЕмма 5. Для любого нелипиицева модуля непрерывности $\omega(\delta)$ в $A_{2}(D) \cap C(\bar{D})$ имеется функиия $f(z)=\varphi_{0}(z)+\bar{z} \varphi_{1}(z)$, равная 0 на $\partial D$, для которой $\omega(f, \bar{D}, \delta) \leqslant$ $\omega(\delta), \delta \geqslant 0$, в то время как голоморфные функиии $\varphi_{0}(z)$ и $\varphi_{1}(z)$ почти ни в одной точке $\zeta \in \partial D$ не имеют угловых пределов.

ДокАЗАТЕльСтво. По теореме Барта и Шнейдера [6] для любой функции $p(r)>0$, $0 \leqslant r<1$, монотонно возрастающей к $+\infty$ с ростом $r$ от 0 до 1 , сушествует голоморфная в $D$ функция $\varphi(z) \neq 0$, имеющая почти в каждой точке $\zeta \in \partial D$ радиальный предел 0 , причем $|\varphi(z)|<p(|z|), z \in D$. По теореме единственности Лузина-Привалова [7] $\varphi(z)$ не имеет угловых пределов почти нигде на $\partial D$. Голоморфные компоненты функции $f(z):=(1-z \bar{z}) \varphi(z)$ также почти нигде на $\partial D$ не имеют угловых пределов. Пусть при $0<r<1$ функция $p(r)$ такова, что

$$
24(1-r) p(r)<\omega(1-r) \quad \text { и } \quad p\left(1-\frac{1}{2}(1-r)\right)<2 p(r) .
$$

Тогда при $z \in D$ имеем

$$
|f(z)|<2(1-|z|) p(1-(1-|z|))<\frac{1}{12} \omega(1-|z|),
$$


$f(z) \rightarrow 0$ при $|z| \rightarrow 1$. Положим $f(z)=0$ на $\partial D$. Если $z \in \bar{D}$ и $t \in \partial D$, то для $\Delta:=f(t)-f(z)$ получаем

$$
|\Delta| \leqslant \frac{1}{12} \omega(1-|z|) \leqslant \omega(|z-t|) .
$$

Если $z, t \in D$ и, например $1-|t|<|t-z|$, то $1-|z| \leqslant(1-|t|)+|t-z|$ и

$$
|\Delta| \leqslant|f(t)|+|f(z)| \leqslant \frac{1}{12}(\omega(1-|t|)+\omega(1-|t|)+\omega(|t-z|))<\omega(|t-z|) .
$$

Пусть теперь $z, t \in D,|t-z| \leqslant 1-|z| \leqslant 1-|t|$. Если функция $g(z)$ голоморфна в некоторой области $G$, то при $2 d=\rho=: \rho(z, \partial G)$ и $\mathscr{C}:=\{t:|t-z|=d\}$ имеем

$$
\left|g^{\prime}(z)\right|=\left|\frac{1}{2 \pi i} \int_{\mathscr{C}} g(t)(t-z)^{-2} d t\right| \leqslant \frac{2}{\rho} \max \left\{|g(t)|: t \in G, \rho(t, \partial G)=\frac{\rho}{2}\right\} .
$$

Отсюда $\left|\varphi^{\prime}(z)\right|<p(1-d) / d$, и для приращения $d f$ функции $f$ вдоль бесконечно малого вектора $d z$ получаем неравенство

$$
|d f| \leqslant\left(\left|\frac{\partial f}{\partial x}\right|+\left|\frac{\partial f}{\partial y}\right|\right)|d z|<2\left(2 p(1-2 d)+\frac{2 \cdot 2 d \cdot p(1-d)}{d}\right)|d z| \leqslant 12 p(1-d)|d z| .
$$

Интегрирование $d f(z)$ вдоль отрезка $[z, t]$ дает неравенство

$$
|\Delta| \leqslant 12 p(1-d)|t-z| \leqslant 24|t-z| p(1-|t-z|) \leqslant \omega(|t-z|) .
$$

Итак, $|f(t)-f(z)| \leqslant \omega(|t-z|)$ для любых $z, t \in \bar{D}$, что и требовалось.

4. О теоремах типа Харди-Литтлвуда. Хорошо известны следующие две теоремы Харди и Литтлвуда (см., например, [8, гл. IX]).

Теорема I. Голоморфная в единичном круге $D$ функиия $f$ удовлетворяет в $D$ условию $\operatorname{Lip} p, 0<p \leqslant 1$, тогда и только тогда, когда $\left|f^{\prime}(z)\right|=O\left((1-|z|)^{p-1}\right)$, $|z| \rightarrow 1$.

Tеорема II. Пусть $f$ голоморфна в $D$ и непрерьвна на $\bar{D}$. Тогда если $f \in \operatorname{Lip} p$ на $\partial D, 0<p \leqslant 1$, mо $f \in \operatorname{Lip} p$ ина $\bar{D}$.

Обобщающее теорему II неравенство Тамразова из [5] упоминалось вьше. Следующая теорема непосредственно обобщает теорему I на полианалитические функции и произвольные $p \neq 0$.

ТЕоремА 2. В области $G$, ограниченной простым или составным жордановым аналитическим контуром, имеем импликачии

$$
\begin{gathered}
f \in \mathfrak{M}^{p}(G) \Longleftrightarrow \frac{\partial f}{\partial z} \in \mathfrak{M}^{p-1}(G) \quad u \quad \frac{\partial f}{\partial \bar{z}} \in \mathfrak{M}^{p-1}(G), \quad p \in \mathbb{R} \backslash\{0\}, \\
f \in \mathfrak{M}^{p}(G) \Longrightarrow \frac{\partial f}{\partial z} \in \mathfrak{M}^{p-1}(G) \quad u \quad \frac{\partial f}{\partial \bar{z}} \in \mathfrak{M}^{p-1}(G), \quad p \in \mathbb{R},
\end{gathered}
$$

а также импликачии, получающиеся из них заменой либо $\mathfrak{M}$ на $C U$, либо $\partial f / \partial z$ и $\partial f / \partial \bar{z}$ на $\partial f / \partial x$ u $\partial f / \partial y$, либо обеими этими заменами. 
ДоказАтельство. Пусть сначала $f$ голоморфна в $G, f \in \mathfrak{M}^{p}(G)$. Если $p \leqslant 0$, то $\left|f^{\prime}(z)\right|=O\left(\rho^{p-1}\right)($ см. $(25)), f^{\prime} \in \mathfrak{M}^{p-1}(G)$. Если $0<p<1$, то по теореме I $f^{\prime} \in \mathfrak{M}^{p-1}(G)$. Отсюда $f \in \mathfrak{M}^{p}(G) \Longrightarrow f^{\prime} \in \mathfrak{M}^{p-1}(G)$ и для любого нецелого $p>0$. Для целых $p>0$ эта импликация следует из ограниченности $f^{(p)}(z)$.

Пусть теперь $f \in A_{n}(G) \cap \mathfrak{M}^{p}(G), \Gamma$ - один из связных контуров, составляющих $\partial G$. Тогда $f_{k}(z):=(\bar{z}-a(z))^{k} h_{k}(z)$ (см. (5)) также принадлежат классу $\mathfrak{M}^{p}$ вблизи $\Gamma$ (теорема 1). Так как $h_{k} \in \mathfrak{M}^{p-k}$ вблизи $\Gamma$, то $h_{k}^{\prime} \in \mathfrak{M}^{p-k-1}$, и $\partial f_{k} / \partial z, \partial f_{k} / \partial \bar{z} \in \mathfrak{M}^{p-1}$ вблизи $\Gamma$ (см. (4)). Импликация (27) доказана. Если $\partial f_{k} / \partial z, \partial f_{k} / \partial \bar{z} \in \mathfrak{M}^{p-1}(G)$ и $p \neq 0$, то в силу леммы $3 f \in \mathfrak{M}^{p}(G)$, так что импликация (26) также доказана.

СлЕДСтвиЕ 6. Для того чтобы полианалитическая в единичном круге D функция $f$ удовлетворяла на $\bar{D}$ условию $\operatorname{Lip} \alpha, 0<\alpha \leqslant 1$, необходимо и достаточно, чтобь выполнялось

$$
\left|\frac{\partial f}{\partial z}\right|+\left|\frac{\partial f}{\partial \bar{z}}\right|=O\left((1-|z|)^{\alpha-1}\right) .
$$

Что касается теоремы II, то уже для бианалитических функций в общем случае дело обстоит совсем иначе: нетрудно построить функцию $f \in A_{2}(D) \cap C(\bar{D})$, равную 0 всюду на $\partial D$ и имеющую на $\bar{D}$ сколь угодно плохой модуль непрерьвности. По-видимому, в случае аналитичности $\partial G$ порядковое равенство $\omega(f, \bar{G}, r) \asymp \omega(f, \Gamma, r)$ выполняется для любой функции $f \in A_{n}(G) \cap C(\bar{G}), n \geqslant 2$, тогда и только тогда, когда справедлива следуюшая теорема единственности:

$$
f \in A_{n}(G) \cap C(\bar{G}) \text { и } f(z)=0 \quad \forall z \in \partial G \Longrightarrow f(z) \equiv 0 .
$$

Области $G$, в которых справедлива эта теорема, полностью описаны в [9] (при $n=2)$ и в [10] (при $n \geqslant 3$ ).

5. Возможные обобщения. В приложениях возникает необходимость в локальных формах теорем 1 и 2 и их следствий, т.е. в формулировках для случая, когда $\Gamma$ - некоторая достижимая простая открытая аналитическая дуга границы области $G$. Такие обобщения получить нетрудно. Необходимые для этого естественные обобщения определения 2 сделать легко. Например, ограниченность (равномерная непрерьвность) порядка $p \in(-\infty,+\infty)$ функции $f$ вблизи дуги $\Gamma \subset \partial G$ будет означать наличие для

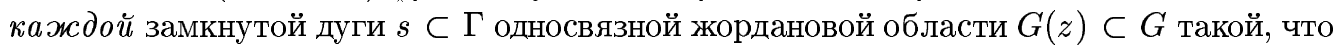
$s \subset \partial G(s), f \in \mathfrak{M}^{p}(G(s))$ (соответственно $f \in C U^{p}(G(s))$ ). Справедливость локальных форм лемм $2-4$, теорем 1,2 и их следствий означает, что эти предложения вьполняются в некоторой области $G(s)$ указанного типа для каждой замкнутой дуги $s \subset \Gamma$.

Определение функции Шварца $a_{\Gamma}(z)$ для простой открытой аналитической дуги $\Gamma$ строится так:

а) возьмем какую-либо открытую дугу $S \subset G$ так, чтобы ее концы совпадали с концами $J, K$ дуги $\Gamma$ и дуга $S$ вместе с $\Gamma, J$ и $K$ ограничивала односвязную подобласть $g$ области $G$;

б) возьмем какое-либо конформное отображение $w=\varphi(z)$ области $g$ на единичньй круг $D$, переводящее точки $J$ и $K$ в некоторые точки $j=\varphi(J)$ и $k=\varphi(K)$ окружности $\partial D$, симметричные относительно оси $O x(k=\bar{j})$;

в) функцию $a_{\Gamma}(z)$ для дуги Г определим по $\varphi$ равенством (3). 
Из этого определения следует, что $a_{\Gamma}(z)$ голоморфна и однолистна в некоторой окрестности дуги $\Gamma$, а уравнение этой дуги записьвается в виде $\bar{z}=a_{\Gamma}(z)$. Имея функцию Шварца $a=a_{\Gamma}$, представляем вблизи $\Gamma$ функцию $f, n$-аналитическую в $G$, в виде $(5)$ (или в виде (2), если $G \subset D, \Gamma \subset \partial D)$, после чего доказательства локальных форм указанных лемм, теорем и следствий практически полностью сводятся к доказательствам, приведенным выше.

Оценки модулей непрерывности компонент и их производных нетрудно получить, используя леммы 1,2 и соответствующее обобщение леммы 4 (см. доказательство теоремы 1).

С помощью методов, примененных в работе, наши результаты обобшаются на функции, полигармонические в круге $D$ (как известно, такие функции $f$ можно представить в виде $(2)$, где $g_{k}$ - гармонические функции в $D$, а $P(z, \bar{z})=Q(x, y)$ - полином от $x, y)$. Такие обобщения можно получить и из самих теорем 1 и 2 , принимая во внимание, что $f n$-аналитична тогда и только тогда, когда $\operatorname{Re} f n$-гармонична (cм. (2)), и что $\operatorname{Re} g_{k} \in \mathfrak{M}^{p} \Longleftrightarrow g_{k} \in \mathfrak{M}^{p}, \operatorname{Re} g_{k} \in C U^{p} \Longleftrightarrow g_{k} \in C U^{p}\left(g_{k}\right.$ голоморфна в $\left.D, p \neq 0\right)$.

\section{СПИСОК ЦИТИРОВАННОЙ ЛИТЕРАТУРЫ}

[1] Balk M. B. Polyanalytic Functions. Math. Res. V. 63. Berlin: Akademie-Verlag, 1991.

[2] Davis P. J. The Schwarz Function and Its Applications. Carus Math. Monographs. V. 17. Washington (D.C.): Math. Assoc. America, 1974.

[3] Mathurin C. Fonction caracteristique d'un contour algebrique simple. Application a l'equation $\Delta(\Delta \varphi)=0$ de l'elastostatique plane. Notes techniques du Ministere de l'Air. V. 105. Paris: Service de Documentation et d'Information technique de l'Aeronautique, 1962.

[4] Долженко Е.П. Об особых точках непрерьвных гармонических функций // Изв. АН СССР. Сер. матем. 1964. Т. 28. №6. С. 1251-1270.

[5] Тамразов П. М. Контурные и телесные структурные свойства голоморфных функций комплексного переменного // УМН. 1973. Т. 28. №1. С. 131-161.

[6] Barth K.F., Schneider W.J. On the impossibility for extending the Riesz uniqueness theorem to functions of slow growth // Ann. Acad. Sci. Fenn. Ser. A. I Math. 1968. V. 432. P. 1-9.

[7] Привалов И. И. Граничные свойства аналитических функций. М.-Л.: ГИТТЛ, 1950.

[8] Голузин Г.М. Геометрическая теория функций комплексного переменного. М.: Наука, 1966.

[9] Хоп Н. Т. О нормальной разрешимости задачи Дирихле для одной эллиптической системы // Дифференц. уравнения. 1966. Т. 2. № 2. С. 214-225.

[10] Расулов К. М. О решении краевых задач типа задачи Дирихле для полианалитических функций // Докл. АН СССР. 1989. Т. 309. №6. С. 1309-1313. 